\title{
Geometric Phase of a Quantum Dot System in Nonunitary Evolution
}

\author{
Sun Yin* and D. M. Tong ${ }^{\dagger}$ \\ Department of physics, Shandong University, Jinan, 250100, P.R. China
}

(Dated: November 6, 2018)

\begin{abstract}
Practical implementations of quantum computing are always done in the presence of decoherence. Geometric phase is useful in the context of quantum computing as a tool to achieve fault tolerance. Recent experimental progresses on coherent control of single electron have suggested that electron in quantum dot systems is promising candidate of qubit in future quantum information processing devices. In this paper, by considering a feasible quantum dot model, we calculate the geometric phase of the quantum dot system in nonuitary evolution and investigate the effect of environment parameters on the phase value.
\end{abstract}

PACS numbers: 73.21.La,03.67.Lx

\footnotetext{
*yinsun@sdu.edu.cn

$\dagger$ tdm@sdu.edu.cn
} 
The quantal geometric phase was first discovered by Berry [1] in 1984 in considering the quantum systems under cyclic adiabatic evolution. It has aroused much attention of researchers due to its importance. Since then the original notion of Berry phase has been extended to a general concept of geometric phase for pure states as well as for mixed states. The extension to pure states in nonadiabatic cyclic evolution was developed by Aharonov and Anandan [2] in 1987, and that to pure states in nonadiabatic and noncyclic evolution was done by Samuel and Bhandari [3] in 1988. Further generalizations and refinements, by relaxing the constrains of adiabaticity, unitarity, and cyclicity of the evolution, have since been carried out [4, 5]. While all these extensions are of quantum systems in pure states, Uhlmann [6] was the first to address the geometric phases of mixed states within the mathematical context of purification. A physical definition of geometric phases for mixed states in unitary evolution was put forward by Sjöqvist et al. [7] in 2000 based on quantum interferometry, and it was recast in a kinematic description by Singh et al. [8]. The generalization of mixed geometric phases to quantum systems in nonunitary evolution was given by Tong et al. [9] in 2004. More works on geometric phases related to states for open systems may be seen in Refs. [10].

The geometric property of the geometric phase has stimulated many applications. It has been found that the geometric phase plays important roles in quantum phase transition, quantum information processing, etc. [11]. The geometric phase shift can be fault tolerant with respect to certain types of errors, thus several proposals using NMR, laser trapped ions, etc. have been given to use geometric phase to construct fault-tolerant quantum information processer [12], and the fault-tolerant geometric quantum computation gate has been demonstrated in experiments using NMR [13].

Geometric phase is useful in the context of quantum computing as a tool to achieve fault tolerance. Practical implementations of quantum computing are always done in the presence of decoherence. Fortunately, recent experimental progresses on coherent control of single electron have suggested that electron in quantum dot systems is a promising candidate of qubit in future quantum information processing devices [14], because it has long spin coherence time. This start us to investigate the geometric phase of quantum dot systems in nonuitary evolution. In this paper, we calculate the geometric phase of a feasible quantum dot model and investigate the effects of the environment parameters to the phase value.

The model is illustrated as Fig,1. Two quantum dots, $\mathrm{QD}_{1}$ and $\mathrm{QD}_{2}$, are coupled to each 


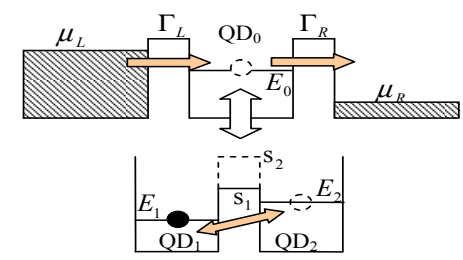

FIG. 1: Illustration of the model.

other with strength $s_{1}$. An electron is trapped in the quantum dots and it tunnels between the two quantum dots. Only one energy level is considered in each quantum dot, and hence the electron and the two quantum dots construct a two-level quantum system, a qubit. The environment of the system consists of another quantum dot, $\mathrm{QD}_{0}$, and two leads connecting to $\mathrm{QD}_{0}$. The left lead has higher chemical potential than the right lead. Electrons can tunnel from the left lead to $\mathrm{QD}_{0}$ and then tunnel out to the right lead. For simplicity, we assume that only one electronic state with the energy level $E_{0}$ in $\mathrm{QD}_{0}$ is correlated and $\mu_{L}>E_{0}>\mu_{R}$, where $\mu_{L}$ and $\mu_{R}$ are the chemical potentials of the left lead and the right lead respectively. Once there is an electron in $\mathrm{QD}_{0}$, it will affect the coupling between $\mathrm{QD}_{1}$ and $\mathrm{QD}_{2}$ by changing the coupling strengthes from $s_{1}$ to $s_{2}$. This is an interesting model, of which the relaxation and decoherence and quantum measurement have been well studied [15, 16]. The model is easily performed in experiment, and it may play a potential selection for geometric quantum computation of using quantum dot systems.

Noting that the qubit system, comprising the trapped electron and the two dots, is an open system being in mixed state, we use the formula of geometric phases for mixed states in nonunitary evolution given in Ref. [9]. For an open quantum system, described by the reduced density operator, $\rho(t)=\sum_{k=1}^{2} \omega_{k}(t)\left|\phi_{k}(t)\right\rangle\left\langle\phi_{k}(t)\right|, t \in[0, \tau]$ the geometric phase is given by the formula,

$$
\gamma(\tau)=\operatorname{Arg}\left(\sum_{k=1}^{2} \sqrt{\omega_{k}(0) \omega_{k}(\tau)}\left\langle\phi_{k}(0) \mid \phi_{k}(\tau)\right\rangle e^{-\int_{0}^{\tau}\left\langle\phi_{k}(t) \mid \dot{\phi}_{k}(t)\right\rangle d t}\right),
$$

where $\omega_{k}(t)$ is the $k$-th eigenvalue of the reduced density matrix, $\left|\phi_{k}(t)\right\rangle$ is the corresponding eigenvector, and $\tau$ is the total evolutional time.

In order to calculate the geometric phase of the qubit system, we need to obtain the 
reduced density operator. The Hamiltonian of the large system can be expressed as

$$
\begin{aligned}
H & =H_{s}+H_{e}+H_{i}, \\
H_{s} & =E_{1} a_{1}^{\dagger} a_{1}+E_{2} a_{2}^{\dagger} a_{2}+s_{1}\left(a_{1}^{\dagger} a_{2}+a_{2}^{\dagger} a_{1}\right), \\
H_{e} & =E_{0} c_{0}^{\dagger} c_{0}+\sum_{l} E_{l} c_{l}^{\dagger} c_{l}+\sum_{r} E_{r} c_{r}^{\dagger} c_{r}+\sum_{l, r}\left(\Omega_{l} c_{l}^{\dagger} c_{0}+\Omega_{r} c_{0}^{\dagger} c_{r}+\text { H.c. }\right), \\
H_{i} & =\left(s_{2}-s_{1}\right) c_{0}^{\dagger} c_{0}\left(a_{1}^{\dagger} a_{2}+a_{2}^{\dagger} a_{1}\right) .
\end{aligned}
$$

Here, $H_{s}, H_{e}, H_{i}$ are the Hamiltonians corresponding to the system itself, the environment and the interaction between the system and its environment, respectively; $a_{1}^{\dagger}$ and $a_{2}^{\dagger}\left(a_{1}\right.$ and $a_{2}$ ) are the electron creation (annihilation) operators in the two quantum dots; $c_{l}^{\dagger}$ and $c_{r}^{\dagger}\left(c_{l}\right.$ and $c_{r}$ ) are the electron creation (annihilation) operators in the environment corresponding to the left lead and the right lead respectively; $E_{1}$ and $E_{2}$ are the energy level of $\mathrm{QD}_{1}$ and $\mathrm{QD}_{2} ; \Omega_{l}\left(\Omega_{r}\right)$ is the coupling parameter of left (right) lead with the quantum dot $\mathrm{QD}_{0}$. For simplicity, we have considered electrons as spinless fermions, and we have used $E_{l}, E_{r}, \Omega_{l}$, and $\Omega_{r}$ to represent $E_{L l}, E_{R r}, \Omega_{L l}$, and $\Omega_{R r}$ respectively.

The wave function of the large system, $|\Psi(t)\rangle$, satisfies the Schrödinger equation, $i \frac{d|\Psi(t)\rangle}{d t}=$ $H(t)|\Psi(t)\rangle$. The reduced density operator $\rho(t)$ may be expressed as the partial traces of $|\Psi(t)\rangle\langle\Psi(t)|$ with respect to the environment consisting of the quantum dot $\mathrm{QD}_{0}$ and the two leads, $\rho(t)=\operatorname{tr}_{D_{0}} \varrho(t)$, where $\varrho(t)=\operatorname{tr}_{L s}|\Psi(t)\rangle\langle\Psi(t)|$. Following the method used in Refs. [16], we may get the equations of motion for the elements of density matrix $\varrho(t)$. The bases of $\varrho(t)$ consists of four discrete states, $|1\rangle \equiv|1,0,0\rangle,|2\rangle \equiv|1,0,1\rangle,|3\rangle \equiv|0,1,0\rangle$, $|4\rangle \equiv|0,1,1\rangle$, where $\left|n_{1}, n_{2}, n_{3}\right\rangle$ means that there are $n_{1}, n_{2}, n_{3}$ electrons in $\mathrm{QD}_{1}, \mathrm{QD}_{2}$, $\mathrm{QD}_{0}$ respectively. In the approximation of constant density of states, let $\Gamma_{L}=2 \pi\left|\Omega_{L}\right|^{2} \rho_{L}$ and $\Gamma_{R}=2 \pi\left|\Omega_{R}\right|^{2} \rho_{R}$, where $\rho_{L}\left(\rho_{R}\right)$ is the density of states for the left (right) lead, and $\Omega_{L}$ $\left(\Omega_{R}\right)$ denotes the constant coupling parameter $\Omega_{l}\left(\Omega_{r}\right) . \Gamma_{L}\left(\Gamma_{R}\right)$ depicts the tunneling rate between the left (right) lead and $\mathrm{QD}_{0}$. In this case, the elements $\varrho_{i j}$ of the density matrix 
$\varrho(t)$ satisfy[17],

$$
\begin{aligned}
& \dot{\varrho}_{11}=-\Gamma_{L} \varrho_{11}+\Gamma_{R} \varrho_{22}-i s_{1}\left(\varrho_{13}-\varrho_{31}\right), \\
& \dot{\varrho}_{22}=-\Gamma_{R} \varrho_{22}+\Gamma_{L} \varrho_{11}-i s_{2}\left(\varrho_{24}-\varrho_{42}\right), \\
& \dot{\varrho}_{33}=-\Gamma_{L} \varrho_{33}+\Gamma_{R} \varrho_{44}-i s_{1}\left(\varrho_{31}-\varrho_{13}\right), \\
& \dot{\varrho}_{44}=-\Gamma_{R} \varrho_{44}+\Gamma_{L} \varrho_{33}-i s_{2}\left(\varrho_{42}-\varrho_{24}\right), \\
& \dot{\varrho}_{13}=-i \epsilon_{0} \varrho_{13}-i s_{1}\left(\varrho_{11}-\varrho_{33}\right)-\Gamma_{L} \varrho_{13}+\Gamma_{R} \varrho_{24}, \\
& \dot{\varrho}_{24}=-i \epsilon_{0} \varrho_{24}-i s_{2}\left(\varrho_{22}-\varrho_{44}\right)-\Gamma_{R} \varrho_{24}+\Gamma_{L} \varrho_{13} .
\end{aligned}
$$

The initial condition is taken as $\left.\varrho_{i j}\right|_{t=0}=1$, for $i=j=1$, or 0 , for all other $i, j$, corresponding to the case that the electron is in $\mathrm{QD}_{1}$ and no electron is in $\mathrm{QD}_{0}$. Here $\epsilon_{0}=E_{1}-E_{2}$, is the energy difference of the energy levels of $\mathrm{QD}_{1}$ and $\mathrm{QD}_{2}$. The elements $\rho_{i j}$ of the reduced density matrix $\rho(t)$ of qubit can be then expressed as

$$
\rho_{11}=1-\rho_{22}=\varrho_{11}+\varrho_{22}, \rho_{12}=\rho_{21}^{*}=\varrho_{13}+\varrho_{24}
$$

Once the reduced density matrix is obtained, we can calculate its eigenvalues $\omega_{k}(t)$ and eigenvectors $\left|\phi_{k}(t)\right\rangle$, and we have

$$
\begin{aligned}
& \omega_{1,2}(t)=\frac{1 \pm \sqrt{\left(\rho_{11}-\rho_{22}\right)^{2}+4\left|\rho_{12}\right|^{2}}}{2} \\
& \left|\phi_{1}(t)\right\rangle=\frac{1}{\sqrt{1+\frac{\left|\rho_{12}\right|^{2}}{\left(\omega_{1}-\rho_{22}\right)^{2}}}}\left[\begin{array}{c}
1 \\
\frac{\rho_{21}}{\omega_{1}-\rho_{22}}
\end{array}\right], \\
& \left|\phi_{2}(t)\right\rangle=\frac{1}{\sqrt{1+\frac{\left|\rho_{12}\right|^{2}}{\left(\omega_{2}-\rho_{11}\right)^{2}}}}\left[\begin{array}{c}
\frac{\rho_{12}}{\omega_{2}-\rho_{11}} \\
1
\end{array}\right] .
\end{aligned}
$$

The initial condition taken above implies $\omega_{1}(0)=1, \omega_{2}(0)=0$, and $\left|\phi_{1}(0)\right\rangle=$ $\left[\begin{array}{l}1 \\ 0\end{array}\right], \quad\left|\phi_{2}(0)\right\rangle=\left[\begin{array}{l}0 \\ 1\end{array}\right]$.

The evolution of the system can be illustrated by the path traced in Bloch sphere. The three-dimensional coordinates in the Bloch sphere are $x=\rho_{12}+\rho_{21}, y=i\left(\rho_{12}-\rho_{21}\right)$, and $z=\rho_{11}-\rho_{22}$, respectively. By using the Four-order Runge-Kutta method, we may numerically resolve the differential equations in (3) and obtain the value of the density operator. Fig. 2 shows the path traced by the state of the system, where the parameters are chosen as $\Gamma_{L}=1.0, \Gamma_{R}=2.0, s_{1}=1.0, s_{2}=0.5, \epsilon_{0}=-2.0$. Hereafter, we take 
the parameter $s_{1}$ as the base unit. All the other parameters with energy dimension, such as $\Gamma_{L}, \Gamma_{R}, s_{2}$, are measured by the unit $s_{1}$, and the time is measured by $1 / s_{1}$. As the time goes on, the path starts from $(0,0,1)$, which corresponds to the state that the trapped electron is in $\mathrm{QD}_{1}$, and moves spirally to $(0,0,0)$, which corresponds to the state that the electron has half probability in $\mathrm{QD}_{1}$ and half in $\mathrm{QD}_{2}$.

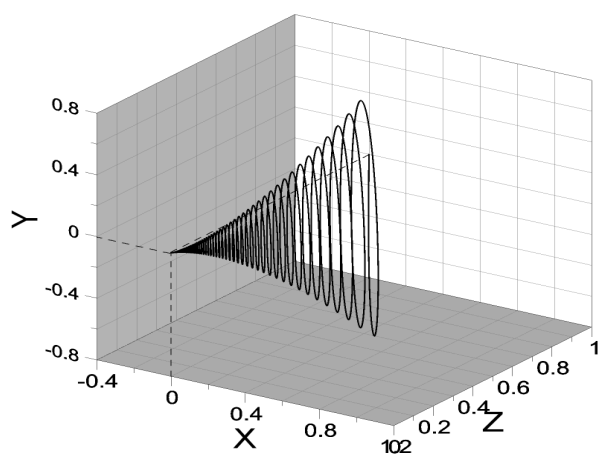

FIG. 2: The Bloch sphere of the density matrix.

Substituting Eq. (5) into Eq. (1), we can calculate the geometric phase of the system. It may be simply expressed as $\gamma(\tau)=i \int_{0}^{\tau}\left\langle\phi_{1}(t) \mid \dot{\phi}_{1}(t)\right\rangle d t$. To sketch out the changing tendency of the geometric phase, we numerically calculate the geometric phase. The parameters are again chosen as $\Gamma_{L}=1.0, \Gamma_{R}=2.0, s_{1}=1.0, s_{2}=0.5, \epsilon_{0}=-2.0$. The result is shown as Fig. 3, The geometric phase is usually put in region $[0,2 \pi)(\bmod 2 \pi)$. In order to show

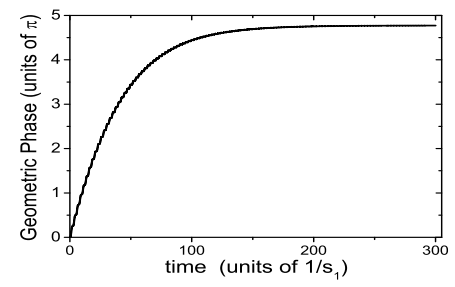

FIG. 3: The geometric phase as a function of time.

entirely the changing tendency of the phase and express clearly the path dependence of the geometric phase, here we give the schematic by using the calculated values without making a $2 \pi$-modulus. The recast of the results in $[0,2 \pi)$ is trivial.

From Fig. 3, we find that the geometric phase is changing as the time is going on, and it finally saturates to a constant value. The saturation value is a characteristic value for a given configuration of parameters, which may be simply called as the characteristic geometric 
phase (CGP). This is consistent with the 'geometricity' of the geometric phase, that is, the geometric phase is only dependent on the path traced by the state of the system, but not on the dynamics. When evolutional time is small, the spiral path has large spiral radius and the changing of the path is notable, and thus the changing of the geometric phase is obvious too. With evolutional time going on, the spiral radius of the path becomes small and the changing rate of the spiral path are reduced, and therefore the changing of the geometric phase will be reduced too. The system will finally evolves to the point $(0,0,0)$, and from then on the path will be little changing, and so does the geometric phase.

In the model, there are three environment parameters $s_{2}, \Gamma_{L}, \Gamma_{R}$. We now investigate the effects of these parameters on the phase values. For this, we will consider two kinds of geometric phase values, the geometric phase corresponding to the whole evolutional time, i.e., the CGP, and the geometric phase corresponding to a special time interval $T$.

Firstly, we observe the effect of the parameters on the CGP. Fig. 4(a) shows the effect
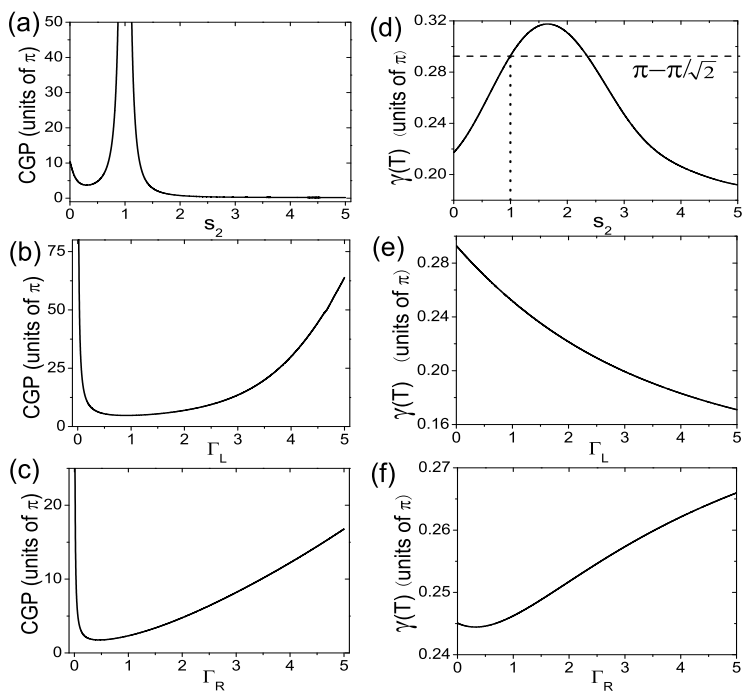

FIG. 4: The geometric phases, CGP and $\gamma(T)$, as functions of the parameters $s_{2}, \Gamma_{L}$, and $\Gamma_{R}$. The parameters except for the one taken as variable are chosen as $\Gamma_{L}=1.0, \Gamma_{R}=2.0, s_{1}=1.0, s_{2}=$ $0.5, \epsilon_{0}=-2.0$.

of $s_{2}$ on CGP. From the figure, we see that CGP is strongly dependent on the parameter $s_{2}$. Specially, CGP is infinitely large at $s_{2}=s_{1}$. This is a reasonable result, because $s_{2}=s_{1}$ means that the environment does not affect the qubit system. In the case, the qubit is in the pure state, which is evolving repeatedly along a closed circle in the Bloch sphere, and 
CGP will accumulate infinitely as the time is going on. However, as the parameter $s_{2}-s_{1}$ is becoming large from zero, the value of the phase will reduce. The phase values will approach to zero when $s_{2}-s_{1}$ is large enough. This may be explained by the following argument. The larger $s_{2}-s_{1}$ means the larger correlation between the environment and the qubit system, which leads to the smaller spiral radius of the path traced by the state of the system. When the environments' effect is stronger enough, the path may approaches to a line directly from $(0,0,1)$ to $(0,0,0)$ and the corresponding geometric phase will be near to zero.

Figs. 4(b) and (c) show the effect of parameters $\Gamma_{L}$ and $\Gamma_{R}$ on CGP. From the figures, we find that the two curves in the figures are similar. CGP becomes infinitely large at $\Gamma_{L}=0$ or $\Gamma_{R}=0$, and it is also approaching to infinity as $\Gamma_{L}$ or $\Gamma_{R}$ is going to large values. These observations are consistent with the physical construction in the model, as we have taken $\Gamma_{R}=2$ in Fig. 4 (b) and $\Gamma_{L}=1$ in 4 (c). Roughly speaking, when $\Gamma_{L}$ is small and $\Gamma_{R}$ is large, electrons are hard to tunnel into $\mathrm{QD}_{0}$ from the left lead but easy to tunnel out of $\mathrm{QD}_{0}$. There is nearly no electron staying in $\mathrm{QD}_{0}$ in all the time, i.e., the coupling between $\mathrm{QD}_{1}$ and $\mathrm{QD}_{2}$ is mainly $s_{1}$. The effect of the environment on the qubit is negligible, and the qubit may be taken as a closed two-level system with coupling strength $s_{1}$. The picture of CGP corresponding to the case is the left part of Fig. 4(b) or the right part of 4(c). When $\Gamma_{L}$ is large and $\Gamma_{R}$ is small, electrons are easy to tunnel into $\mathrm{QD}_{0}$ from the left lead but hard to tunnel out of $\mathrm{QD}_{0}$. There is an electron staying in $\mathrm{QD}_{0}$ almost in all the time, i.e., the coupling between $\mathrm{QD}_{1}$ and $\mathrm{QD}_{2}$ is dominated by $s_{2}$. The effect of the environment on the qubit is only to change the coupling strength between $\mathrm{QD}_{1}$ and $\mathrm{QD}_{2}$ from $s_{1}$ to $s_{2}$, and the qubit system may be taken as a closed system but with coupling $s_{2}$. The picture corresponding to this case is the right part of the curve in Fig. 4(b) or left part of the curve in Fig. 4(c). When $\Gamma_{L}$ and $\Gamma_{R}$ are in the same order, the qubit is an open system in mixed state. The path traced by the mixed state is a spiral curve and so corresponds to finite values of CGP.

Secondly, we observe the effect of the parameters on the geometric phase for the special time interval $T$. If there is no coupling between the qubit and the environment, or $s_{2}=s_{1}$, the qubit system will be in a pure state and it will evolve from the initial state $(0,0,1)$ back to itself after a time interval $T$, making up a closed circle in the Bloch sphere. In the case where $\epsilon_{0}=-2$ and $s_{1}=1$, we have $T=\pi / \sqrt{2}$, and the geometric phase corresponding to the closed cycle is $\gamma(T)=\pi-\pi / \sqrt{2}$. However, if $s_{2} \neq s_{1}$, the path traced by the state in the 
Bloch sphere will become an unclosed curve and the geometric phase $\gamma(T)$ will be changed under the effect of the environment. Therefore, $\gamma(T)$ may be used to describe the effect of the environment on geometric phase in an finite time, during which the pure state evolves one circle. Figs. $4(\mathrm{~d}), 4(\mathrm{e})$ and $4(\mathrm{f})$ show the effect of parameters $s_{2}, \Gamma_{L}$ and $\Gamma_{R}$ on $\gamma(T)$, respectively. The curves in the figures may be explained by applying a similar discussion as above.

In conclusion, we have calculated the geometric phase of a feasible quantum dot model and investigate the effects of the environment parameters to the phase value. Here, we not only presented the parameters's effect on the characteristic geometric phase, which corresponding to the whole evolutional time, but also studied their effect on the geometric phase in a finite time interval $T$, defined by using pure state without the effect of environment. The approach of calculating the geometric phase in the paper is reliable. While the other approaches of defining the geometric phase of open systems have met criticisms [18], the kinematic approach used in the paper has been widely applied to investigate the open systems in various environments [19]. Our investigation on geometric phase is helpful to completely understand the properties of the quantum dot system.

This work is supported by NSF of China under Grant Nos.10675076, 10875072 and 10804062.

[1] M.V. Berry, Proc. R. Soc. London Ser. A 392, 45 (1984).

[2] Y. Aharonov and J. Anandan, Phys. Rev. Lett. 58, 1593 (1987); J. Anandan and Y. Aharonov, Phys. Rev. D 38, 1863 (1988).

[3] J. Samuel and R. Bhandari, Phys. Rev. Lett. 60, 2339 (1988).

[4] N. Mukunda and R. Simon, Ann. Phys. (N.Y.) 228, 205 (1993).

[5] A.K. Pati, Phys. Rev. A 52, 2576 (1995); J. Phys. A 28, 2087 (1995).

[6] A. Uhlmann, Rep. Math. Phys. 24, 229 (1986); Lett. Math. Phys.21, 229 (1991).

[7] E. Sjöqvist et al., Phys. Rev. Lett. 85, 2845 (2000).

[8] K. Singh et al., Phys. Rev. A 67, 032106 (2003).

[9] D.M. Tong et al., Phys. Rev. Lett. 93, 080405 (2004).

[10] M. Ericsson et al., Phys. Rev. A 67, 020101(R) (2003); A. Carollo et al., Phys. Rev. Lett. 90, 
160402 (2003); K. P. Marzlin, S. Ghose, and B.C. Sanders, ibid. 93, 260402 (2004); X. X. Yi, L. C. Wang, and T. Y. Zheng, ibid. 92, 150406 (2004).

[11] A. Bohm et al., The Geometric Phase in Quantum Systems (Springer, New York, 2003); S.-L. Zhu, Phys. Rev. Lett. 96, 077206 (2006); A.C.M. Carollo and J.K. Pachos, ibid. 95, 157203 (2005).

[12] G. Falci et al., Nature 407, 355 (2000); X.-B. Wang and M. Kerji, Phys. Rev. Lett. 87, 097901 (2001); L.-M. Duan, J.I. Cirac and P. Zoller, Science 292, 1695 (2001).

[13] J.A. Jones et al., Nature 403, 869 (2000).

[14] D. Loss and D.P. DiVincenzo, Phys. Rev. A 57, 120 (1998); J.R. Petta et al., Science 309, 2180 (2005); D. Press et al., Nature 456, 218 (2008); P. San-Jose et al., Phys. Rev. B 77, 045305 (2008).

[15] T.M. Stace et al., Phys. Rev. B 70,205342 (2004).

[16] S.A. Gurvitz and D. Mozyrsky, Phys. Rev. B 77, 075325 (2008); S.A. Gurvitz and G.P. Berman, ibid. 72, 073303 (2005); T. Gilad and S.A. Gurvitz, Phys. Rev. Lett. 97, 116806 (2006).

$[17]|\Psi(t)\rangle$ may be expanded in the picture of the creation and annihilation operators $\left(a_{1}^{\dagger}, a_{1}, a_{2}^{\dagger}, a_{2}, c_{l}^{\dagger}, c_{l}, c_{r}^{\dagger}, c_{r}\right)$ applying on the "vacuum" state of the large system with all the levels in the two leads being filled with electrons up to the Fermi levels. Substituting the expansion of $|\Psi(t)\rangle$ into the Schrödinger equation, one may get the differential equations satisfied by the expansion amplitudes, from which Eq.(3) can be derived by tracing out the freedoms of the two leads. Please refer to [16] for details.

[18] A. Bassi and E. Ippoliti, Phys. Rev. A 73, 062104 (2006); M. Ericsson et al., Phys. Rev. Lett. 91, 090405 (2003).

[19] X.X. Yi, L.C. Wang and W. Wang, Phys. Rev. A 71, 044101 (2005); X.X. Yi et al., ibid. 73, 052103 (2006); A.T. Rezakhani and P. Zanardi, ibid. 73, 052117 (2006); F.C. Lombardo and P.I. Villar, ibid. 74, 042311 (2006); X.X. Yi and W. Wang, ibid. 75, 032103 (2007); J. Dajka, M. Mierzejewski and J. Luczka, J. Phys. A 41, 012001 (2008); J. Dajka and J. Luczka, ibid. 41, 442001 (2008); S. Banerjee and R. Srikanth, Euro. Phys. J. D 46, 335 (2008). 